\title{
ENVEJECIMIENTO COGNITIVO Y PROCESAMIENTO DEL LENGUAJE: CUESTIONES RELEVANTES
}

\author{
COGNITIVE AGING AND LANGUAGE PROCESSING: \\ RELEVANT ISSUES
}

\author{
MÓNICA VÉLIZ \\ BERNARDO RIFFO \\ Universidad de Concepción. Concepción, Chile. \\ meveliz@udec.cl,bernardo@udec.cl
}

\section{BEATRIZ ARANCIBIA}

Universidad Católica de la Santísima Concepción. Concepción, Chile.

barancib@ucsc.cl

\section{RESUMEN}

¿Qué sucede con el lenguaje cuando se envejece? ¿Se produce una declinación de las habilidades lingüísticas? ¿Cambian los patrones de desempeño de los mayores si se los compara con los jóvenes? ¿Qué variables pueden explicar los cambios que se producen? Para dar respuesta a estas y otras interrogantes se presentan las teorías más revelantes que se han desarrollado para dar cuenta de los efectos del envejecimiento cognitivo en los procesos de comprensión y producción del lenguaje, a saber: enlentecimiento, déficit inhibitorio, déficit de transmisión, reducción de la memoria operativa. Se hace también una revisión de los hallazgos más relevantes de la investigación psicolingüística de orientación gerontológica. El examen abarca todos los niveles de procesamiento y considera los fenómenos de comprensión y de producción vinculados a la palabra, la oración y el discurso. El análisis del problema se completa presentando aportes provenientes de la neuropsicología y discutiendo cuestiones críticas relacionadas con los métodos de investigación que se usan en el área y el papel que juegan los factores culturales y sociales.

Palabras claves: Procesamiento del lenguaje, envejecimiento cognitivo, memoria operativa.

* Este artículo forma parte del proyecto Fondecyt 1050591 desarrollado por el Grupo de Investigación en Psicolingüística Experimental de la Universidad de Concepción. 


\begin{abstract}
What happens to language when humans age? Is there a decline in the language abilities in old age? Do performance patterns in the elderly change when compared to those of younger individuals? What variables can explain these changes? In order to answer these and other questions, the authors present the most relevant theories developed to explain age-related cognitive effects in language comprehension and production processes, for instance slowing, inhibitory deficit, transmission deficit, working memory decline. Furthermore the most notable findings of gerontology-based psycholinguistic research are reviewed, covering all language processing levels. It also takes into account the language comprehension and production phenomena regarding word, sentence and discourse levels. The problem analysis is completed with contributions from neuropsychology as critical issues are discussed related to search methods used in the area and the role played by social and cultural factors.
\end{abstract}

Keywords: Language processing, cognitive aging, working memory.

Recibido: 06-04-2009. Aceptado: 29-01-2010.

\title{
1. INTRODUCCIÓN
}

- 1 estudio del envejecimiento cognitivo y su efecto sobre los procesos de comUprensión y producción del lenguaje se ha constituido en las últimas décadas en un tópico de investigación de alto interés para la psicolingüística, tanto de orientación descriptiva como experimental.

Ello se explica -en parte- por la trascendencia que pueden tener tales estudios mirados desde una perspectiva social y demográfica. En un escenario en donde los promedios de vida de la población han aumentado dramáticamente y el porcentaje de personas que viven más allá de los 60 años ha crecido y sigue creciendo de manera notable, trazar de manera nítida las diferencias entre el envejecimiento normal y el patológico así como entender la naturaleza y extensión del deterioro que la vejez puede causar en el lenguaje deviene una cuestión crítica, tanto si lo que se quiere es proyectar el potencial intelectual de los adultos mayores en la sociedad futura o definir las condiciones que puedan ayudar a minimizar los efectos del deterioro a que se ha aludido.

Pero más allá de la sensibilidad de los investigadores ante los cambios sociales, el envejecimiento cognitivo se constituye en un territorio de investigación interesante para la psicolingüística en la medida que abre la posibilidad de someter a prueba empírica las teorías y modelos del procesamiento del lenguaje en un contexto diferente, cual es el del cerebro envejecido. El contacto entre ambas áreas ha resultado enriquecedor y dado origen a un dominio de estudio muy productivo $\mathrm{y}$ 
promisorio. Factor fundamental ha sido el hecho de que el lenguaje sea un campo de investigación teórica y metodológicamente bien desarrollado de la ciencia cognitiva y se cuente con modelos bien especificados de la organización del sistema lingüístico.

\section{UNA MIRADA AL PROCESAMIENTO DEL LENGUAJE}

La habilidad para comunicarse mediante el lenguaje es -sin duda- fundamental en la vida humana. Entender y producir mensajes orales o escritos de manera rápida y precisa es de importancia vital para obtener información del ambiente, realizar exitosamente las actividades que conforman la vida diaria y mantener relaciones sociales normales. Y, aunque esta habilidad parezca llevarse a cabo sin esfuerzo alguno, es en realidad el resultado de un complejo conjunto de procesos sensoriales y cognitivos. La tarea de la psicolingüística es justamente explicar la naturaleza y funcionamiento de esos procesos que subyacen a la comprensión y producción del lenguaje.

La idea general que se ha impuesto es que se trata de funciones mentales complejas constituidas por un conjunto integrado de procesos y representaciones que operan en diversos niveles. Para entender cómo funciona este complejo sistema hay que saber cuáles son sus componentes, qué estructura los organiza, de qué naturaleza son las operaciones psicolingüísticas que cada componente del sistema cumple y cómo se ensamblan unos con otros en el curso temporal de la acción. Se suele hablar de la arquitectura funcional del lenguaje para referirse al conjunto de estos niveles de procesamiento.

Si se sitúa el foco de atención en los procesos de comprensión, habrá que pensar en mecanismos que den cuenta del procesamiento ortográfico y fonológico, del procesamiento léxico, del procesamiento sintáctico y semántico de las oraciones y del procesamiento en el nivel del discurso. Para cada uno de esos mecanismos habrá que concebir algoritmos particulares que describan paso a paso las operaciones atinentes a su función particular.

Lo anterior implica proponer modelos explicativos que den cuenta de cómo el sistema cognitivo humano se las arregla para transformar la señal lingüística en una representación fonológica de la palabra, cómo es que puede reconocer las palabras y activar y seleccionar un significado para cada una, cómo procede para analizar las secuencias de palabras y otorgarles una estructura sintáctica, cómo construye el significado de cada oración, cómo opera para integrar todos esos significados en una representación unificada del significado del texto. A ello hay que agregar una teorización acerca de cómo el comprendedor mantiene activa la información en su memoria operativa cuando debe establecer referencia o decidir entre significados alternativos o cómo recupera información de su memoria de largo plazo para ha- 
cer inferencias cuando en el curso del procesamiento ello se requiere.

Si el fenómeno en estudio fueran los procesos de producción del lenguaje, el camino que habría que recorrer sería diferente, pero de igual modo que en la comprensión se encontrarían procesos de gran complejidad.

La discusión en torno a la arquitectura computacional del sistema de procesamiento del lenguaje -orientada por los paradigmas dominantes de la ciencia cognitiva: el simbólico-computacional y el conexionista e inspirada en las concepciones modular e interactiva del sistema- ha sido muy prolífica y generado una infinidad de modelos teóricos vinculados con los distintos niveles de procesamiento. Exponer detalladamente todos los modelos, sin embargo, no es el propósito de este artículo. Nuestro objetivo se orienta más bien a dar cuenta de cómo la psicolingüística ha intentado hasta ahora responder las interrogantes fundamentales que surgen en torno al problema de la relación entre procesamiento del lenguaje y envejecimiento. En concreto, nos proponemos examinar las principales teorías que se han desarrollado para explicar el envejecimiento cognitivo (Salthouse, 1996), determinar qué aspectos del lenguaje -y en qué medida- se ven afectados por esta condición, cuál es el papel de la memoria en este proceso, qué paradigmas metodológicos han dominado el campo de investigación y cuáles se proyectan en el futuro inmediato, y, finalmente, establecer qué sabemos sobre la relación entre el envejecimiento del cerebro y el procesamiento del lenguaje.

\section{TEORÍAS DEL ENVEJECIMIENTO COGNITIVO Y EL LENGUAJE}

\subsection{Enlentecimiento}

Tal vez uno de los fenómenos mejor establecidos en relación con el envejecimiento cognitivo sean las diferencias observadas en la velocidad de procesamiento cuando se compara el desempeño de jóvenes y mayores frente a diferentes tareas. Los datos empíricos son claros: los viejos son más lentos que los jóvenes. En nuestras propias investigaciones sobre procesamiento sintáctico (Riffo y Véliz, 2008), hemos encontrado en forma sistemática tiempos de lectura más altos en los adultos mayores que los adultos jóvenes en oraciones con diferente estructura sintáctica.

Según la teoría del enlentecimiento (Cerella, 1985; Salthouse, 1996), la declinación en el desempeño cognitivo se produciría por un enlentecimiento general de la velocidad de transmisión neural. Respaldan este enfoque hallazgos empíricos que muestran que las medidas de velocidad motora-perceptiva comparten mucha de la varianza asociada a la mayor edad en el desempeño de un amplio rango de tareas cognitivas (Junqué y Jodar, 1990).

Aplicada al procesamiento del lenguaje, la teoría del enlentecimiento puede dar cuenta de la mayor dificultad que experimentan los senescentes, por ejemplo, en 
la comprensión del habla rápida. ¿Qué explicación tiene ello en el marco de la teoría? Como se sabe, la velocidad de procesamiento es crítica en el habla porque la señal lingüística es continua y se despliega en el tiempo. Un procesamiento rápido es esencial para reconocer en forma correcta los fonemas a partir de una onda sonora cuyos componentes varían rápida y constantemente y cuyas claves acústicas transmiten información en paralelo sobre segmentos sucesivos (Liberman, Cooper, Shankweiler, \& Studdert-Kennedy, 1967). Una ilustración adecuada del fenómeno la proporciona el tiempo de inicio de la sonoridad o VOT (voice onset time), rasgo que permite distinguir, por ejemplo, la secuencia /ba/ de /pa/ en español. Si el procesamiento es más lento que lo habitual, la señal se desvanece antes de que se alcance a producir la discriminación del carácter abierto o cerrado de la consonante.

La lentitud puede resultar negativa también cuando en el curso del procesamiento se requiere que haya disponibilidad simultánea de información (Carpenter, Miyaki \& Just, 1994; Salthouse, 1996). El problema surge cuando la información proveniente de un proceso temprano ha decaído en el momento en que se la requiere para procesar información posterior en la secuencia. Ello puede ocurrir en el proceso implicado en la comprensión de una oración. La construcción del significado global de la oración se verá afectada si el procesamiento es tan lento que el significado de las palabras iniciales ha decaído antes de que se procesen semánticamente las palabras finales.

\subsection{Déficit inhibitorio}

Esta teoría sostiene que el envejecimiento debilita los procesos inhibitorios que regulan la atención sobre los contenidos de la memoria operativa. Ello afecta a una amplia gama de actuaciones cognitivas entre las cuales se incluyen la comprensión y producción del lenguaje (Hasher \& Zacks, 1988; Zacks \& Hasher, 1997). La memoria, según esta teoría, no solo debe activar y procesar ciertas unidades de información, sino también debe filtrar los contenidos no pertinentes para que no se produzca interferencia o confusión. Para ello deben operar simultáneamente dos funciones: una función de acceso, que restringe el ingreso de información irrelevante a la memoria operativa y una función de supresión, que elimina la información que ha dejado de ser útil o que estuvo en estado de latencia y finalmente no fue utilizada. En conjunto, ambas contribuyen a que las representaciones mentales que alcanzan el umbral de activación sean coherentes y se articulen con las metas que guían el procesamiento. Si estos mecanismos inhibitorios de la atención se debilitan, puede suceder que mientras se está procesando la información surjan pensamientos intrusivos o se hagan asociaciones no relevantes, elementos que, al competir por recursos de procesamiento, reducen la capacidad funcional de la memoria de trabajo afectando la comprensión o el recuerdo (Zacks, Hasher \& Li, 2000). 
La teoría del déficit inhibitorio se ha aplicado al procesamiento del lenguaje de los adultos mayores para explicar, entre otras cosas, por qué la actuación se ve afectada por estímulos distractores durante la lectura o la audición. Así, Carlson y sus colaboradores (Carlson, Hasher, Zacks \& Connelly, 1995) informan sobre una lectura desproporcionadamente lenta en sujetos mayores cuando enfrentan textos en que se ha introducido material distractor, el cual fue controlado por los experimentadores cuidando que el contenido del elemento distractor se relacionara con el significado textual y su localización no fuera predecible. Por otra parte, Zacks \& Hasher (1997) han utilizado esta teoría para explicar los casos en que la investigación reporta verbosidad en el discurso de las personas mayores y frecuentes alejamientos del tópico central en sus conversaciones.

\subsection{Disminución de la capacidad de la memoria operativa}

Es una teoría bastante influyente cuya hipótesis fundamental postula que a consecuencia del envejecimiento, la memoria operativa -entendida como un sistema de memoria de recursos limitados, dotado al mismo tiempo de capacidad para almacenar y manipular información- experimenta una disminución en su capacidad de trabajo, hecho que impone limitaciones a la habilidad de las personas mayores para comprender o producir oraciones semántica o sintácticamente complejas (Carpenter et al., 1994; Just \& Carpenter, 1992; Kemper \& Kemptes, 1999).

Construir una representación mental del significado de una oración implica asignar una estructura sintáctica y semántica a una secuencia de palabras. Este proceso, dado el carácter lineal de la señal lingüística, exige que se mantengan activos en la memoria los resultados intermedios y finales de las computaciones que se realizan mientras el procesador va analizando secuencialmente las palabras de entrada y construyendo en línea las estructuras sintácticas y semánticas necesarias para la comprensión de la oración. En todo este proceso, los recursos operacionales de la memoria son indispensables. Lo mismo puede afirmarse en el caso de la producción de oraciones. Se requieren recursos de memoria sea para planificar el contenido o generar las ideas, mantener activas las representaciones gramaticales, fonológicas u ortográficas durante la codificación y mantener el control ejecutivo de todo el proceso.

La hipótesis de una declinación de la habilidad de procesamiento sintáctico asociada a la disminución de la capacidad de memoria operativa ha recibido respaldo empírico de numerosos trabajos, especialmente los de Susan Kemper y su equipo (Cheung \& Kemper, 1992; Kemper, 1987; Kemper, Greiner, Marquis, Prenovost \& Mitzner, 2001; Kynette \& Kemper, 1986). En esos estudios, los sujetos mayores obtienen tasas más bajas de desempeño que los jóvenes en tareas que requieren comprensión o recuerdo inmediato de oraciones de estructura sintáctica compleja. Se entiende por ello, por ejemplo, oraciones con incrustación 
múltiple o con incrustación a la izquierda, oraciones relativas objeto, oraciones agramaticales, oraciones ecuacionales, oraciones ambiguas y otras. Muestran también estos estudios una tendencia general de los mayores a producir oraciones gramaticalmente más simples cuando escriben o participan en conversaciones o son entrevistados. Estos resultados han sido interpretados como indicadores de esa declinación. En este contexto, la capacidad de la memoria operativa se ha medido de la manera tradicional usando básicamente la prueba de amplitud de memoria de Daneman y Carpenter (1980).

Hoy día, sin embargo, la hipótesis de la declinación sintáctica está en debate. Dos enfoques con distintas concepciones acerca de la memoria operativa compiten en el área: la llamada teoría de la capacidad o teoría de recurso único de memoria (Just \& Carpenter, 1992; King \& Just, 1991) y la teoría de los recursos de memoria divididos (Caplan \& Waters, 1999). Nos referiremos brevemente a ambas.

El modelo de memoria verbal propuesto por Just y Carpenter (1992), muy cercano al ejecutivo central de Baddeley (1986), sostiene que la comprensión del lenguaje involucra recursos de procesamiento y almacenamiento, que una fuente única de recursos limitados sirve a ambas actividades y que hay diferencias individuales importantes en la capacidad funcional de la memoria operativa. La teoría predice que una capacidad de memoria operativa baja reduce los recursos disponibles para el procesamiento sintáctico haciéndolo menos eficiente. Empíricamente está avalada por correlaciones positivas entre medidas de capacidad de memoria y medidas de eficacia del procesamiento sintáctico (Daneman y Merikle, 1996). En el marco de esta teoría, se predice que la disminución de la capacidad de memoria debida al envejecimiento tendrá como consecuencia una pérdida en la habilidad de procesamiento sintáctico.

La propuesta de Waters y Caplan (1999), en cambio, se basa en una distinción entre procesos interpretativos y post-interpretativos en la comprensión. Defiende la hipótesis de que el procesamiento de la oración depende no de un solo conjunto sino de dos conjuntos de recursos de memoria separados: uno, especializado en el proceso interpretativo inmediato de la oración, que es rápido e involuntario, ocurre en línea y permite reconocer palabras, acceder al léxico y analizar sintáctica y semánticamente la oración; y otro, dedicado a los procesos post-interpretativos, que son los que tienen lugar fuera de curso y se vinculan con el uso de la información obtenida para responder preguntas, emitir juicios de gramaticalidad y otras tareas. Contrariamente a la teoría de la capacidad, la teoría de recursos separados predice que no hay gran efecto de la amplitud de memoria en las tareas de procesamiento sintáctico asociadas a la mayor edad. Hay también datos experimentales (Clifton, Traxler, Mohamed, Williams, Morris \& Rayner, 2003; Waters \& Caplan, 1999) que apoyan el enfoque. Según se reporta en los estudios citados no se encuentra interacción entre capacidad de memoria y la eficiencia con que se realiza 
el análisis sintáctico. Ambas capacidades serían independientes.

Un tema interesante en el debate es la tensión que se genera entre las metodologías on-line o en curso y las off-line o fuera de curso. Como observan Waters y Caplan (1999), las evidencias de declinación sintáctica asociadas a la memoria operativa han sido reportadas principalmente por estudios que usan métodos offline. Los resultados encontrados en esos estudios no podrían atribuirse a declinación sintáctica determinada por la capacidad disminuida de la memoria operativa. Corresponden más bien -según los autores- a dificultades para llevar a cabo tareas que requieren que se ejecute alguna operación en la oración una vez que ha sido interpretada, como cuando se responde a una pregunta o se da un juicio de gramaticalidad, por ejemplo. Consistentes con este planteamiento, son los resultados de estudios que usan metodologías on-line (seguimiento de los movimientos oculares o técnicas de ventana móvil), en los que no se encuentran suficientes evidencias de una relación causal entre disminución de la memoria operativa y dificultad de procesamiento sintáctico (Caplan \& Waters, 1999; Kemmer, Coulson De Ochoa, \& Kutas, 2004; Kemper \& Kemptes, 1999; Kemptes \& Kemper, 1997; Waters \& Caplan, 1999; Waters \& Caplan, 2001).

\subsection{Déficit de transmisión}

La teoría del déficit de transmisión (Burke, MacKay \& James, 2000) se basa en un modelo conexionista localista que concibe el lenguaje como una vasta red de conexiones entre unidades organizadas en un subsistema fonológico y un subsistema semántico. Se postula que muchos de los cambios cognitivos asociados al envejecimiento podrían deberse al debilitamiento de las conexiones entre las representaciones en la memoria. Cuando la fuerza de esas conexiones se debilita, se reduce también la transmisión de la excitación que las prepara para la activación, un fenómeno que los especialistas llaman priming. La transmisión de la excitación puede ser tan reducida que resulte insuficiente para que las representaciones conectadas se activen, proceso necesario para gatillar la recuperación de la información codificada en esas representaciones.

Aunque los déficits de transmisión asociados al envejecimiento están distribuidos a través de todo el sistema de representaciones, los efectos funcionales que pueda producir el déficit dependen de las características de la arquitectura del sistema lingüístico. Así, por ejemplo, el sistema semántico que se caracteriza por conexiones redundantes y convergentes entre las representaciones resulta menos vulnerable a los déficits de transmisión. Así lo sugieren los datos sobre procesamiento semántico existentes, los que muestran que los mayores tienen representaciones semánticas tan ricas como los jóvenes (Ackerman \& Rolfhus, 1999). Diferente es la situación en el sistema fonológico, donde las conexiones entre las unidades son singulares y por ello más vulnerables a los déficits de transmisión. Un buen ejem- 
plo es el fenómeno conocido como "punta de lengua" ${ }^{1}$-muy frecuente entre los mayores- y que se explica justamente por una falla en la recuperación fonológica de la palabra que se quiere producir (Burke, MacKay, Wordslay \& Wade, 1991).

\subsection{Déficit sensorio-perceptivo}

La teoría del déficit sensorio-perceptivo o de la señal degradada (Baltes \& Lindenberger, 1997) plantea que la declinación de los procesos sensoriales y perceptivos asociada al envejecimiento - principalmente, audición y visión- produce entradas erróneas o incompletas en las computaciones de bajo nivel implicadas en la codificación fonológica y ortográfica. Como consecuencia de esta falla se produciría daño en el proceso de selección léxica y en otros procesos subsiguientes (Burke \& Shafto, 2008; Schneider, 2001). No está todavía muy claro en qué medida las pérdidas que pudieran ocurrir en los niveles más altos de la cognición vinculada al lenguaje podrían atribuirse solamente al efecto de señales de entrada degradadas. Hay acuerdo, sin embargo, en que las declinaciones en los procesos de percepción influyen en la comprensión y producción del lenguaje (McDowd, 2001).

El trabajo desarrollado por el equipo de Baltes (Berliner Alterstudie, BASE) ha considerado además un conjunto amplio de variables en relación con el envejecimiento. Así, el enfoque multidimensional adoptado permite vincular los cambios que experimenta el ser humano a medida que avanza en años con factores como las capacidades intelectuales, la personalidad y las relaciones sociales, entre otros. Los resultados de numerosas investigaciones realizadas por el grupo alemán muestran interesantes tendencias, como, por ejemplo, que la correlación entre la edad y los aspectos cognitivos es alta y explica hasta en un 35\% la variación individual, siendo esta relación tal que, a mayor edad, hay un mayor deterioro de habilidades cognitivas, las que se explican por el proceso de degenerativo de todo el soporte biológico de la cognición, especialmente, del sistema nerviosos y perceptivo (audición, visión), como se señaló antes (Smith \& Baltes, 1996). También resulta interesante que la correlación entre la edad y otras dimensiones como la personalidad y las relaciones sociales sea distinta. Los autores consideran que el envejecimiento exitoso requiere una capacidad adaptativa importante, la que se manifiesta a través de mecanismos compensatorios y de regulación de diferentes dominios psicológicos. Dentro de este esquema general, sin embargo, los aspectos perceptivos aparecen como los de mayor peso a la hora de explicar el desempeño en tareas de índole intelectual.

\footnotetext{
${ }^{1}$ Como se explica más adelante, este fenómeno consiste en que el sujeto puede disponer del concepto y de sólo una parte de la representación fonológica de la palabra, por lo que no puede pronunciarla.
} 


\subsection{Modelo de procesamiento autorregulado del lenguaje}

Por último, nos referiremos a un modelo propuesto recientemente por Elizabeth Stine-Morrow y colegas, el modelo de procesamiento autorregulado del lenguaje. La novedad de este modelo se sintetiza en dos hechos: primero, introduce la autorregulación cognitiva como un componente central del procesamiento del lenguaje y, segundo, integra en la explicación del fenómeno el proceso de envejecimiento cognitivo (Miles \& Stine-Morrow, 2004; Stine-Morrow, 2007; Stine-Morrow, Shake, Miles \& Noh, 2006b; Stine-Morrow, Miller \& Hertzog, 2006a).

La autorregulación, estudiada a menudo bajo la rúbrica de metacognición, se entiende como la habilidad para monitorear y controlar los propios procesos cognitivos entre los que se cuentan el conocimiento, la memoria, el aprendizaje, la comprensión del lenguaje, la actuación diestra, el logro de objetivos y otros (Metcalfe \& Kornell, 2003; Nelson, 1996; Peronard, 1997; 1999; Thiede \& Dunlosky, 1999) e incluye decisiones relativas a la asignación de esfuerzos y atención, selección de estrategias de procesamiento, emisión o aplazamiento de respuestas y velocidad a la que la tarea debería ser completada. El modelo, en este contexto, propone una conexión entre los déficits cognitivos producidos por el envejecimiento y los procesos autorreguladores.

Los supuestos básicos son que los mecanismos de autorregulación implicados en el procesamiento del lenguaje operan con los mismos heurísticos que, se postula, dirigen la autorregulación en los procesos del aprendizaje en general, a saber: retroalimentación negativa, reducción de la discrepancia, región de aprendizaje próximo y otros, y que los mecanismos que los sujetos mayores usan para autorregularse en el ámbito del procesamiento del lenguaje experimentan cambios dinámicos a partir de la mediana edad. La evolución que experimentan estos heurísticos refleja procesos de adaptación en aspectos relevantes de la cognición y de la afectividad (Stine-Morrow et al., 2006b).

Un aspecto que ha atraído especialmente la atención de los estudiosos es el cambio que se observa en los patrones de asignación de recursos de procesamiento - por ejemplo, de los tiempos de lectura- en función de distintas tareas y en relación con distintos niveles de procesamiento. Si se asume que la declinación de la capacidad de procesamiento asociada al envejecimiento reduce la efectividad con que se llevan a cabo determinadas computaciones durante la comprensión y, como consecuencia, el tiempo disponible para ser asignado a dichas computaciones se ve limitado, podría pensarse que las diferencias de desempeño -comprensión, memoria o aprendizaje de textos- no se atribuirían sólo a déficits en la capacidad de procesamiento sino también a cambios producidos en los procesos de autorregulación, lo que en términos más concretos podría entenderse como procesos no efectivos de asignación de recursos.

Pero no sólo pueden producirse fallas en la autorregulación, los cambios en el 
comportamiento observados en los adultos mayores pueden considerarse también como evidencias de estrategias de compensación frente a la reducción de la eficacia del procesamiento del lenguaje y del funcionamiento cognitivo en general (Radvansky, Zwaan, Curiel \& Copeland, 2001; Wingfield \& Stine-Morrow, 2000). Mantener el control autorregulador vía mecanismos de selección y compensación parece ser clave para una vejez exitosa (Baltes, 1997; Fernández-Ballesteros, 2005).

Un componente relevante en el modelo de procesamiento autorregulado del lenguaje es la percepción que tienen los individuos de sus propias capacidades cognitivas. Es frecuente que los adultos mayores no tengan una visión de sus posibilidades de desempeño ajustada exactamente a los hechos. Suele ocurrir, en efecto, que los sujetos, confiando erradamente en su capacidad, tomen decisiones no adecuadas en la asignación de recursos, esto es, decisiones no concordantes con las exigencias de la tarea o con los objetivos planteados. Puede suceder también que se llegue a una pérdida del control de los procesos a partir de una percepción que subestima las verdaderas capacidades dando lugar a cuadros afectivos de ansiedad y otros que impactan negativamente en el desempeño (Stine-Morrow et al., 2006b). No menos importante que la autopercepción son los cambios dinámicos que a lo largo del ciclo vital va sufriendo el individuo en términos de la relación entre sus objetivos y los valores de referencia. Ambos aspectos, por constituir factores determinantes en el modelo teórico, han sido considerados como variables de investigación y, por tanto, objeto de manipulación experimental (Stine-Morrow et al., 2006a).

\subsection{Una perspectiva global}

Mirados en conjunto estos modelos constituyen un cuerpo teórico bastante sugerente y con suficiente poder como para explicar aspectos significativos e interesantes de los cambios que opera el envejecimiento en el desempeño lingüístico. No se ha logrado aún, sin embargo, consolidar una teoría general que integre de manera coherente los conceptos, métodos y hallazgos empíricos obtenidos hasta el momento por los investigadores inspirados en los distintos enfoques.

Tampoco se ha producido una articulación entre los investigadores del desarrollo y los del envejecimiento. Los modelos teóricos, los enfoques y metodologías típicamente han surgido y se han desarrollado en forma aislada. A pesar de que hay una continuidad en el desarrollo del lenguaje humano -los niños crecen, se convierten en adultos y finalmente llegan a adultos mayores- no se han explorado todavía en profundidad los puntos de contacto, la posible continuidad que pudiera existir en los mecanismos y procesos que subyacen en el desempeño lingüístico en las distintas etapas de la vida.

Entre los modelos de envejecimiento que hemos presentado hay dos, sin em- 
bargo, que deben ser destacados: el modelo que explica el envejecimiento como un déficit de transmisión y el recientemente propuesto centrado en la autorregulación. Su superioridad sobre los otros modelos radica en que junto con proponer mecanismos explicativos de los cambios -rasgo que comparten todos los modelos- proponen también un modelo de procesamiento del lenguaje y articulan en ese modelo los cambios de comportamiento que se producen en la vejez, explicándolos como parte de la dinámica misma de procesamiento del lenguaje.

En esta perspectiva, el modelo de Stine-Morrow -a nuestro parecer- constituye una propuesta teórica de alto interés que integra comprehensivamente elementos conceptuales provenientes del procesamiento del lenguaje, la cognición autorregulada y el envejecimiento cognitivo, ofreciendo una explicación dinámica y razonable de lo que ocurre cuando las personas mayores se comprometen en tareas de procesamiento del lenguaje.

Los modelos basados en recursos parecen en general menos fuertes y a menudo se les critica por su circularidad, derivada del hecho de que los mismos resultados empíricos que son explicados por una teoría de recursos reducidos -memoria, por ejemplo- sirven también como evidencia para inferir la existencia de una reducción de recursos debida a la vejez (Salthouse \& Craik, 2000).

\section{4. ¿CUÁLES SON LOS EFECTOS MÁS SIGNIFICATIVOS QUE PRODUCE EL ENVEJECIMIENTO EN LA COMPRENSIÓN Y PRODUCCIÓN DEL LENGUAJE?}

Hasta aquí hemos concentrado el esfuerzo en mostrar los lineamientos teóricos más influyentes y de mayor relevancia que respaldan la investigación sobre el lenguaje y el envejecimiento. Es el momento de preguntarnos cuáles son en concreto los cambios más significativos que caracterizan el desempeño lingüístico de las personas mayores. Una manera de responder es haciendo un recorrido por los niveles de procesamiento que se han descrito. Examinemos entonces los cambios más relevantes.

\subsection{Procesamiento fonológico: reconocimiento auditivo y visual de palabras}

Un hecho de todos reconocido es que los adultos mayores pierden la agudeza auditiva y visual y, como lo muestran algunos estudios (Baltes \& Lindenberger, 1997; Salthouse, Hancock, Meinz \& Hambrick, 1996), este deterioro es creciente a partir de los 70 años.

¿Qué impacto tiene esta pérdida en el desempeño lingüístico? Investigaciones de tipo correlacional y experimental ponen de manifiesto que se producen problemas en el reconocimiento del habla. Los mayores con problemas de audición 
rinden menos que los adultos normales en la identificación de estímulos auditivos, lo que abarca desde sílabas a oraciones. Los cambios en el procesamiento auditivo, sin embargo, parecen ir más allá de la agudeza auditiva e involucrar problemas en funciones cognitivas de alto nivel importantes para el reconocimiento del habla. Así, se ha detectado una pérdida de la sincronía auditiva temporal que afecta al sistema nervioso central y periférico, lo que se manifiesta, por ejemplo, en dificultad para discriminar sonidos que difieren en el tiempo de inicio de la sonoridad o VOT (Schneider, Daneman \& Pichora-Fuller, 2002; Schneider \& Pichora-Fuller, 2001).

Se observan también dificultades en los mayores para percibir el habla en ambiente ruidoso, especialmente cuando hay muchas personas hablando al mismo tiempo. Si bien hay acuerdo en que los déficits sensoriales y perceptivos contribuyen a hacer vulnerable el procesamiento en este contexto, hay desacuerdo sobre la importancia relativa que en ello tengan los factores sensoriales y los cognitivos. Algunos autores (Hasher, Lustig \& Zacks, 2007; Tun \& Wingfield, 1999) conceden un peso mayor a los factores cognitivos, entre los que figuran el enlentecimiento, el déficit inhibitorio y la disminución de los recursos de procesamiento.

Los datos de la investigación relacionada con el reconocimiento visual de palabras -área menos estudiada que la relativa al reconocimiento auditivo- revelan, por su parte, que la declinación de la agudeza visual así como otros déficits de procesamiento asociados a los movimientos oculares y la retina afectan la habilidad para leer, especialmente en condiciones vinculadas con baja iluminación y bajo contraste (Schneider \& Pichora-Fuller, 2000). Hay evidencia experimental (Madden, Langley, Denny, Turkington, Provenzale \& Hawk, 2002) de que los déficits sensoriales de los adultos mayores están ligados a una declinación de la activación del córtex visual y ello explicaría las latencias de respuesta más altas que muestran durante el reconocimiento de palabras en tareas de decisión léxica. De igual forma que para el procesamiento auditivo del lenguaje, el procesamiento visual decae en los mayores cuando la percepción tiene lugar en condiciones en que intervienen distractores o "ruido" visual que interfiere en el proceso de lectura (Madden, 1992).

En suma, el reconocimiento auditivo y visual de palabras se ve afectado en la vejez por una declinación en el procesamiento perceptivo. Es tarea de la investigación futura determinar si ello se explica por la degradación de la señal o si debe atribuirse a problemas asociados a los recursos cognitivos disponibles.

\subsection{Producción fonológica}

Un aspecto bien documentado de las pérdidas asociadas al envejecimiento son los déficits que sufren los mayores en la recuperación fonológica (Burke, 1999; Burke et al., 2000; Vitevitch \& Sommers, 2003). La evidencia más fuerte viene 
de la ocurrencia de un estado conocido como "punta de lengua", situación en que temporalmente no se puede producir una palabra muy conocida. Se trata de un fallo típico de acceso léxico que los adultos mayores suelen experimentar con más frecuencia que la deseada. En medio del proceso de "punta de lengua", el afectado sólo puede generar información parcial sobre la fonología de la palabra -el primer fonema, el número de sílabas u otra información. El fenómeno, frecuente en el lenguaje espontáneo pero también inducido en el laboratorio mediante presentación de imágenes, se explica muy bien mediante la teoría del déficit de transmisión, que postula que el envejecimiento reduce la transmisión de excitación a las representaciones en la memoria, en este caso las representaciones fonológicas (Burke et al., 1991; Vitevitch \& Sommers, 2003). La hipótesis de que la experiencia de "punta de lengua" se produce debido a que los mayores son menos capaces de inhibir competidores en el proceso de producción no ha sido corroborada por la investigación (Burke et al., 1991).

El fenómeno del acceso léxico ha sido profusamente investigado en relación con el envejecimiento. Se han sometido a experimentación variables como frecuencia, longitud de las palabras y número de vecinos léxicos, usando preferentemente el priming y el nombrado de imágenes como herramientas de investigación. Los datos obtenidos proporcionan evidencia de un aumento de los fallos de recuperación fonológica en la vejez y son consistentes con la teoría de los déficits de transmisión.

\subsection{Procesamiento en el nivel léxico-semántico}

En contraste con la declinación asociada a los procesos de recuperación fonológica y léxica, hay poca evidencia de cambio en los procesos de recuperación semántica. $\mathrm{Al}$ parecer, las representaciones conceptuales que subyacen al significado del lenguaje a nivel de palabra permanecen bien preservadas en la vejez como lo muestran revisiones realizadas en este dominio (Kemper, 1992; Thornton \& Light, 2006; Wingfield \& Stine-Morrow, 2000, entre otros). Datos experimentales provenientes de estudios de priming semántico en personas mayores confirman que no sólo las representaciones son más fuertes debido a la edad, también las conexiones son más ricas y resistentes a los déficits de transmisión en la red semántica (James \& MacKay, 2001; MacKay \& James, 2001).

Un aspecto destacable es el relacionado con el caudal léxico de las personas de edad mayor. Los estudios sobre envejecimiento reportan por lo general puntajes más altos para los viejos que para los jóvenes en las pruebas estándares de vocabulario (Kemper \& Sumner, 2001; Lindenberger \& Baltes, 1997; Singer, Verhaeghen, Ghisletta, Lindenberger \& Baltes, 2003). El conocimiento léxico-semántico acumulado durante la vida adulta permanece, pues, estable hasta bien avanzada edad. 


\subsection{Procesamiento de oraciones}

Si bien el procesamiento semántico a nivel léxico está preservado en los mayores, hay alguna evidencia de que el procesamiento semántico de las oraciones puede declinar en la vejez debido posiblemente a la lentitud con que se realizan las operaciones encargadas de construir una representación mental del significado. La evidencia proviene de estudios experimentales que emplean técnicas electrofisiológicas - potenciales evocados- para obtener información acerca de la respuesta del cerebro frente a determinados estímulos lingüísticos (Federmeier, McLennan, De Ochoa, \& Kutas, 2002). Un hallazgo de estos estudios es que los adultos mayores usan el contexto oracional con menos efectividad que los jóvenes para activar una palabra cuyo significado sea congruente con esa oración. La explicación es que los mayores requieren más tiempo para que el contexto oracional afecte el procesamiento de una palabra. En nuestros estudios sobre procesamiento de oraciones de estructura compleja hemos encontrado evidencia sostenida de que las personas mayores requieren más tiempo que las jóvenes para responder a preguntas de comprensión (Riffo y Véliz, 2008), lo que es congruente con la hipótesis aludida.

En lo relativo al procesamiento sintáctico de oraciones de estructura compleja, como se recordará, no se ha logrado demostrar una declinación de la habilidad sintáctica de la cual sea responsable la disminución de la capacidad de la memoria operativa.

Aun cuando numerosos estudios muestran, efectivamente, que los adultos mayores tienen tendencia a escribir o producir oralmente oraciones estructuralmente más simples que los jóvenes (Cheung \& Kemper, 1992; Kemper, 1987; Kemper, Thompson, \& Marquis, 2001; Kynette \& Kemper, 1986; Obler, Fein, Nicholas \& Albert, 1991), es dudoso que ello se deba a problemas en la memoria operativa que repercuten en el procesamiento. Las diferencias podrían deberse más bien a factores de orden pragmático (Altmann \& Kemper, 2006; Davidson, Zacks \& Ferreira, 2003; Ferreira \& Patson, 2007).

En tareas de comprensión, los datos son algo controvertidos y las opiniones están divididas. La tendencia que empieza a imponerse es que la habilidad de procesamiento sintáctico está preservada en los adultos mayores y es inmune a las fallas que pueda experimentar la memoria operativa general, pues el sistema de memoria humano dispondría de un componente especializado en el procesamiento en línea de la oración (Caplan \& Waters, 1999; Grossman, Cooke, DeVita, Alsop, Detre, Chen \& Gee, 2002; Waters \& Caplan, 2001). La mayor lentitud que se observa en los adultos mayores cuando procesan en línea oraciones de sintaxis compleja podría tener otras explicaciones (Kemper, Herman \& Liu, 2004; Waters, Caplan, Alpert \& Stanczak, 2003). 


\subsection{Procesamiento del discurso}

Un aspecto esencial de la comprensión del discurso corresponde a la construcción de una representación mental de carácter multidimensional que integra la información extraída del texto con el conocimiento previo: el llamado modelo de situación. Varios estudios muestran que la construcción y uso de modelos de situación durante la comprensión está bien preservada en los adultos mayores (Radvansky et al., 2001; Radvansky, Copeland, Berish, \& Dijkstra, 2003).

Hay evidencias, sin embargo, de que la influencia que los modelos de situación ejercen en el proceso de comprensión es más fuerte en los mayores que en los jóvenes. Ello se manifiesta, por ejemplo, en mejor memoria de los mayores para la información proveniente del modelo de situación que la que viene del texto o en que son más sensibles a la violación del modelo y a efectos de distancia o funcionalidad en las relaciones que se establecen entre los distintos componentes de la situación que se modela (Radvansky et al., 2003).

En cuanto a las estrategias que se usan para asignar los recursos de procesamiento, en términos generales se puede decir que los adultos mayores tienden a priorizar durante la comprensión los aspectos vinculados con la construcción del modelo de situación, esto es, los factores de más alto nivel en el texto, dedicándoles más tiempo y, por ende, más recursos cognitivos (Stine-Morrow, Miller \& Leno, 2001). Los mayores y los jóvenes reciben igualmente la influencia de la información proveniente del texto base o estructura proposicional; pero, mientras los mayores la descartan luego de formar el modelo de situación, los jóvenes la retienen (Stine-Morrow, Morrow \& Leno, 2002).

En suma, la comprensión del discurso en los viejos parece implicar un aumento de la confianza en las estructuras discursivas, como lo es el modelo de situación. El porqué de este comportamiento no parece depender de déficits de recursos de memoria como se había pensado inicialmente sino de cambios en los objetivos y prioridades de las personas de más edad, o sea, de factores pragmáticos.

En cuanto a la producción del discurso, se ha observado que la densidad de las ideas que se generan tiende a declinar como lo muestran, por ejemplo, la escritura de ensayos autobiográficos, la descripción oral de imágenes y otras tareas discursivas (Kemper et al., 2001; Kemper \& Sumner, 2001). La causa de esta caída en la densidad proposicional, asociada fuertemente a la enfermedad de Alzheimer, ha sido estudiada por Snowdon y colegas (Snowdon, Greiner, Kemper, Nanayyakara, \& Mortimer, 1999).

Un comportamiento que suele observarse en los mayores mientras conversan es la verbosidad y habla fuera de tópico, conducta que se ha explicado invocando la teoría del déficit inhibitorio: los mayores tendrían dificultades para inhibir información irrelevante que se genera mientras se está produciendo el discurso (Arbuckle \& Pushkar Gold, 1993). La explicación más aceptada hoy, sin embargo, es 
diferente. No se estaría en presencia de un déficit sino ante un cambio pragmático. Los mayores, al parecer, cambian los objetivos de la conversación enfatizando las narrativas personales y los eventos significativos en sus vidas en lugar de la descripción concisa de hechos (James, Burke, Austin \& Hulme, 1998).

Un tema interesante de investigación vinculado con el discurso es el relativo a las características e impacto que tiene el discurso que va dirigido a los mayores. En inglés se ha acuñado el término elderspeak para designar este registro especial que se emplea para hablar con los mayores como un intento de acomodarse anticipadamente a las dificultades de comunicación, en cierto sentido similar al usado con los niños pequeños (babytalk). Los estudios realizados coinciden en reconocer que el modo como se habla a los ancianos tiene un impacto poderoso en los sentimientos que ellos desarrollan hacia sí mismos y en la percepción que sobre ellos se tiene. Incluso se ha determinado que puede influir en el desempeño frente a tareas cognitivas, mejorando el rendimiento en ciertos casos o empeorándolo en otros. (Kemper \& Harden, 1999; McGuire, Morian, Goldding \& Smyer, 2000). Los rasgos que caracterizan este discurso y su efecto sobre los adultos mayores no han sido abordados en español por especialistas en el discurso.

\section{PROCESAMIENTO DEL LENGUAJE Y ENVEJECIMIENTO DEL CEREBRO}

La investigación psicolingüística -incluida la orientada al estudio del envejecimiento- se ha basado fundamentalmente en métodos conductuales. Es decir, que los datos que deben recogerse para someter a verificación empírica los modelos y teorías se obtienen de medidas de la actuación cognitiva, tales como tiempo de reacción y corrección de la respuesta. El progreso innegable que ha experimentado la psicolingüística en el curso de su historia se ha asentado esencialmente en los métodos conductuales aludidos.

En los últimos años, sin embargo, los métodos electro-fisiológicos y de neuroimagen cerebral -potenciales evocados, resonancia magnética funcional y emisión tomográfica de positrones- han alcanzado significativos avances. Ello ha abierto a los investigadores la posibilidad de obtener información de la actividad cerebral mientras se están desempeñando funciones tales como hablar, leer o comprender y permitido profundizar la comprensión de las relaciones entre el lenguaje, los procesos cognitivos, y las estructuras y funciones cerebrales.

Situándonos ya en la perspectiva del envejecimiento y dado que los procesos cognitivos dependen de la anatomía y fisiología del cerebro, es lógico esperar que los cambios conductuales observados como parte del proceso de envejecer estén ligados a cambios en la integridad de la arquitectura y función cerebral. Este fenómeno corresponde a lo que se denomina envejecimiento cognitivo normal, el 
cual se caracteriza por la ocurrencia de cambios neuroanatómicos, neuroquímicos y metabólicos cuyos efectos comienzan a notarse paulatinamente a contar de los sesenta años, aunque con una gran variabilidad individual. ¿Qué es lo que se ha encontrado?

Estudios en que se emplean medidas de neuroimagen en reposo revelan una disminución de entre un 5 a un $7 \%$ en el volumen cerebral, la cual se asocia principalmente con una pérdida de mielina, lípidos y proteínas; una disminución del volumen de la sustancia gris y del flujo sanguíneo cerebral, y con cambios a nivel neuronal. Estos fenómenos afectan más intensamente a las regiones anteriores del cerebro que a las posteriores, siendo los lóbulos frontales los que muestran una mayor tasa de declinación, especialmente en la zona prefrontal (Buckner, 2004; Escobar, 2001; Gil, 2000; Grady, 2001; Park 2000). Este patrón de envejecimiento cerebral coincide con datos conductuales que muestran un mayor declive de las funciones cognitivas asociadas al funcionamiento de las estructuras de la corteza prefrontal (Buckner, 2004). Entre tales funciones se encuentran operaciones de percepción, atención, reconocimiento de palabras escritas, y procesos de codificación y recuperación de información que requieren la participación de distintos componentes de la memoria. No obstante, los datos tienden a mostrar que no todas las estructuras de la corteza prefrontal son igualmente vulnerables al paso de los años, lo que ayudaría a explicar por qué no todas las funciones cognitivas se ven comprometidas de la misma manera en la vejez normal. Existe además variabilidad individual, por lo que no necesariamente todos los adultos mayores sanos evidencian los efectos de estos cambios de la misma manera o con la misma intensidad.

Otra serie de modificaciones en el funcionamiento cerebral en la vejez la constituyen los cambios a nivel molecular y metabólico que afectan el equilibrio bioquímico del cerebro y entre los cuales se cuentan los relacionados con los neurotransmisores, cuya disponibilidad tiende a disminuir (Buckner, 2004; Gil, 2000). Los especialistas aún no logran describir con precisión cómo interactúa esta disminución de las sustancias responsables de transmitir los impulsos nerviosos durante la sinapsis neuronal con los cambios neuroanatómicos del cerebro. Está establecido, sin embargo, que una restricción de la dopamina, un neurotransmisor relacionado con la regulación de los mecanismos atencionales, y de la acetilcolina, implicada en el funcionamiento de la memoria, influye en el declive de las funciones ejecutivas en la vejez (Buckner, 2004). No es de extrañar, por lo tanto, que las principales teorías acerca del comportamiento cognitivo de los adultos mayores aludan al enlentecimiento de las funciones frontales del cerebro, a problemas con la regulación de la atención y a un declive de la memoria.

Por su parte, estudios basados en neuroimágenes captadas durante la realización de tareas en distintos dominios (v.g. percepción visual, lenguaje, atención, memoria operativa) revelan un enlentecimiento de la respuesta neuroeléctrica así 
como cambios en el patrón de localización y focalización de la actividad neuronal. Este último fenómeno se refiere a una declinación de la zona occipital aparejada con un aumento de la actividad cerebral en la corteza cerebral prefrontal y una mayor bilateralidad hemisférica (Buckner, 2004; Cabeza, 2002; Gil, 2000; Grady, 2001; Grossman et al., 2002; Kemmer, et al., 2004; Park 2000; Park \& Payer, 2001; Payer, Marshuetz, Sutton, Hebrank, Welsh \& Park, 2006). Este patrón de actividad ha sido interpretado como un mecanismo de compensación que viene a suplir las declinaciones cognitivas y sensoriales que se producen en la vejez (Cabeza, 2002). El cerebro viejo recluta estratégicamente recursos neuronales adicionales para mantener la actuación en un alto nivel, lo cual constituye una muestra de la plasticidad y carácter adaptativo de las funciones neurobiológicas del cerebro.

Este enfoque es consistente con evidencia de que la actividad bilateral en los adultos mayores está positivamente correlacionada con desempeño exitoso y se encuentra en los mayores con alto desempeño más que en los de bajo, lo que a su vez parece estar vinculado con factores de carácter más idiosincrásico, asociados al estilo y calidad de vida así como a la práctica continua de las habilidades intelectuales (Polk \& Farah, 1998; Redolat y Carrasco, 1998; Véliz, 2004).

\section{LA INVESTIGACIÓN EN EL ÁMBITO HISPÁNICO}

Al igual que en otras áreas de la investigación, los desarrollos en psicolingüística de orientación gerontológica han cubierto mayoritariamente el inglés como lengua de referencia. Menor ha sido el volumen de investigación en lenguas como el alemán, el holandés y el castellano. En este último caso, no obstante, es posible apreciar algunos equipos de investigadores españoles que ya han consolidado una línea de estudios sobre lenguaje y envejecimiento (Cuetos-Vega, Menéndez-González y Calatayud-Noguera, 2007; Del Río y López-Higes, 2006; Juncos, 1998a; 1998b; Junqué \& Jódar, 1990; entre otros). Por otra parte, en América Latina, aunque se ha destinado poca atención a la problemática objeto de este artículo, existe una interesante actividad emergente en Chile. En el área de comprensión y producción léxica, Echeverría y Urrutia (2004) dan cuenta de una menor velocidad de procesamiento y dificultades para acceder a la información léxica en los adultos mayores, probablemente debido al debilitamiento de las conexiones entre los nodos léxico y fonológico en la vejez. Además, la investigación dio con evidencias de una pérdida de la flexibilidad de las estrategias de búsqueda, lo que podría estar vinculado con fallas de la memoria operativa de largo plazo (LT-WM de Kintsch 1998) o bien con una disminución de la capacidad para inhibir los competidores léxicos que se presentan en las tareas de búsqueda, lo que produciría un bloqueo. En comprensión sintáctica, Véliz (2004), y Riffo y Benoit (2007), analizaron el procesamiento y comprensión de oraciones complejas usando métodos off-line y 
on-line, respectivamente. El primer estudio arrojó un fuerte influjo de factores sociales y culturales que mitigarían o bien acentuarían los efectos del envejecimiento cognitivo, al mismo tiempo que favorecerían o bien limitarían la adquisición y desarrollo de destrezas de memoria a lo largo de la vida, lo que constreñiría la actuación en tareas cognitivas complejas. El segundo estudio mostró un enlentecimiento en el procesamiento interpretativo en los senescentes participantes, y un declive asociado a la edad y la $\mathrm{MO}$ en el procesamiento sintáctico postinterpretativo, aunque con escasa interacción entre las variables $\mathrm{MO}$ y edad.

Otra área que ha tenido algún desarrollo es la investigación sobre producción del discurso. Al respecto, los resultados de Tapia (2005) aportan diversos indicadores que sugieren que con la edad disminuye la capacidad para realizar precisiones o cambios de tópico no previstos. Salazar (2008), por su parte, confirma que en la vejez dicha capacidad experimenta una declinación que, en el caso particular de los ancianos con enfermedad de Alzheimer, es relevante. Por último, Wittig (2004) analiza muestras de relatos orales de senescentes normales y encuentra una incidencia importante del nivel educacional en la estructura de las narraciones; no obstante, los problemas en la construcción del relato no se deberían a un déficit lingüístico, sino a interferencias cognitivas que impiden a los sujetos trasladar satisfactoriamente la representación mental al plano del texto.

\section{CONCLUSIONES}

Los avances en la investigación psicolingüística en el área muestran que no existe una explicación que dé cuenta por sí sola de las diferencias en la actuación lingüística asociadas al envejecimiento. Los datos aportados por nuestras propias investigaciones sugieren, por ejemplo, que un factor de orden cognitivo como la reducción de la capacidad de la memoria operativa resulta insuficiente para explicar el desempeño de los adultos mayores en tareas de procesamiento y comprensión de oraciones. Los datos tienden a mostrar diferencias de orden cualitativo al comparar jóvenes y viejos, con lo cual podríamos estar frente a respaldo empírico de las teorías que sostienen que el cerebro envejecido busca compensar los déficits modificando sus patrones de actividad. Desde el punto de vista de la actuación lingüística, la pregunta que surge es si acaso los adultos mayores hacen uso de estrategias distintas para responder a las demandas de procesamiento del sistema cognitivo. De ser así, éstas podrían responder a un mecanismo de compensación para superar las limitaciones de su sistema de procesamiento. Esta posibilidad abre interrogantes que dan nuevo aliento a la investigación: ‘autorregulan las personas mayores su comportamiento en función de objetivos basados en sus creencias sobre el estado de su sistema cognitivo?, ¿podría igualmente explicarse su desempeño por fallas en los procesos mismos de autorregulación? Otro aspecto interesante 
del problema es averiguar hasta qué punto factores sociales y culturales pueden asociarse a los efectos del envejecimiento en las habilidades lingüístico-comunicativas. No tenemos respuesta todavía para estas interrogantes, pero en su conjunto, se trata-creemos- de un tema de alta relevancia que deberá ser abordado en el futuro con herramientas de investigación experimental más finas que pueden utilizarse para hallar el camino que nos lleve a penetrar poco a poco en ese territorio tan complejo que llamamos mente.

Una síntesis del análisis antes expuesto puede resumirse en los siguientes puntos:

1. Se trata de un campo emergente con más preguntas que respuestas. Si bien es cierto hay ya un par de décadas de investigación, nos encontramos en un momento en que diferentes desarrollos teóricos emergen conformando un campo de gran dinamismo, pero sin que los modelos hayan sido decantados al punto de constituir una explicación cabal. Ello tiene como consecuencia directa que los investigadores realicen su trabajo con más preguntas que respuestas, debido precisamente a que las hipótesis se encuentran en su mayoría en fase de prueba y no se dispone aún de suficiente evidencia empírica.

2. El fenómeno es complejo (multifactorial), ello explica la dificultad teórica para explicarlo a cabalidad con un solo modelo. Envejecer no consiste sólo en la suma de años, se trata de un proceso de alta complejidad, multidimensional, en el que convergen diversos factores y múltiples variables cuyo papel resulta extremadamente difícil de aislar a la hora de efectuar estudios, especialmente de corte experimental. Tal dificultad tiene implicancias teóricas no menores: no es fácil determinar con precisión cuál es la relación entre las variables en juego, por lo tanto, la evaluación de los modelos pierde exactitud y permanecen muchas dudas.

\section{El estudio del envejecimiento lingüistico cognitivo debería abordarse desde una pers-} pectiva evolutiva que integre el proceso de adquisición (completar el ciclo vital). Hasta ahora, los estudios del lenguaje en el adulto mayor se han desarrollado por completo aparte de la investigación en adquisición temprana. La ontogénesis humana es, sin embargo, un proceso continuo que debería ser considerado en su conjunto. Una empresa de esta envergadura demandaría, por cierto, modelos aún más complejos; pero los aportes derivados de tales desarrollos serían, sin duda, altamente interesantes y podrían incluso mostrarnos una visión nueva del fenómeno.

4. El factor sociocultural adquiere mayor relevancia en la investigación, ya que éste es determinante en el desempeño lingüistico de los mayores (incorporarlo a los modelos teóricos). En la medida que el individuo avanza en años, el peso del factor ambiental va incrementándose paulatinamente. Los datos disponibles hasta el momento 
confirman que la historia de cada individuo es determinante para su vejez lingüística, cognitiva y social. Sin embargo, este componente no ha sido incorporado sistemáticamente a los modelos psicolingüísticos del envejecimiento.

5. Aspectos metodológicos: Desde una perspectiva psicolingüistica, junto con los estudios conductuales existentes, se hace necesario el desarrollo de más investigación neurofisiológica. Del mismo modo que en otros campos de la psicolinguística, el desarrollo de la investigación en lenguaje y envejecimiento tiene mucho que esperar de los avances en neurociencias. En efecto, los logros teóricos y, sobre todo, las nuevas tecnologías permitirán obtener imágenes cada vez más precisas del comportamiento del cerebro. Así, se espera disponer de datos más nítidos que permitan orientar mejor la investigación y tomar decisiones más fundamentadas a la hora de evaluar las hipótesis.

6. Los cambios en el desempeño lingüistico asociados al envejecimiento no patológico no constituyen evidencias de un deterioro inhabilitante para la comunicación exito$s a$. Envejecer constituye un proceso global de transformaciones que involucran todas las dimensiones del ser humano; sin embargo, bajo condiciones normales (entendidas como la ausencia de desarrollos patológicos), el organismo realiza operaciones de ajuste a fin de compensar los déficits y mantener un desempeño mínimamente aceptable. En la actuación lingüística de los adultos mayores cognitivamente sanos no hay evidencias de una incapacidad para el uso adecuado del lenguaje en su contexto comunicativo.

\section{REFERENCIAS}

Ackerman, P. L. \& Rolfhus, E. L. 1999. "The locus of adult intelligence: Knowledge, abilities and nonability traits". En Psychology and Aging, 14, pp. 314-330.

Altmann, L. J. P \& Kemper, S. 2006. "Effects of age, animacy and activation order on sentences production". En Language and cognitive processes, 21, pp. 322-354.

Arbuckle, T. Y. \& Pushkar Gold, D. P. 1993. "Aging, inhibition and verbosity". En Journal of Gerontology: Psychological Sciences, 48, pp. 225-232.

Baddeley, A. 1986. Working memory. New York: Oxford University Press.

Baltes, P. B. 1997. "On the incomplete architecture of human ontogenity: Selection, optimization and compensation as foundation of development theory". En American Psychologist, 52, pp. 366-380.

Baltes, P. B. \& Lindenberger, U. 1997. "Emergence of a powerful connection between sensory and cognitive functions across the adult life span: A new window to the study of cognitive aging?". En Psychology and Aging, 12, pp. 12-21. 
Buckner, R. 2004. "Memory and executive funtion in aging and AD: multiple factors that compensate". En Neuron, 44(1), pp. 195-208.

Burke, D. M. 1999. "Language production and aging”. En S. Kemper y R. Kliegl (eds.), Constraints on Language. Aging, Grammar and Memory. Boston: Kluwer Academic Publishers, pp. 3-21.

Burke, D. M. \& Shafto, M. A. 2008. "Language and aging”. En F. I. M. Craik \& T. A. Salthouse (eds.), The handbook of aging and cognition. New York: Psychology Press, pp. 373-443.

Burke, D. M., MacKay, D. G. \& James, L. E. 2000. “Theorical approaches to language and aging". En T. J. Perfect, \& E. A. Maylor (eds.). Models of cognitive aging. New York: Oxford University Press, pp. 204-237.

Burke, D. M., MacKay, D. G, Wordslay, J. S. \& Wade, E. 1991. "On the tip of the tonge: What causes wordfinding failures in jounger and older adults". En Journal of memory and language, 30, pp. 542-579.

Cabeza, R. 2002. "Hemispheric Asymmetry Reduction in Older Adults: The Harold Model". En Psychology and Aging, 17, pp. 85-100.

Caplan, D. \& Waters, G. 1999. "Verbal working memory and sentence comprehension". En Behavioral and Brain Sciences, 22, pp. 77-126.

Carlson, M. C., Hasher, L., Zacks, R. T. \& Connelly, S. L. 1995. "Aging distraction and the benefits of predictable location". En Psychology and Aging, 10, pp. 427-436.

Carpenter, P. A., Miyaki, A. \& Just, M.A. 1994. "Working memory constraints in comprehension: Evidence from individual differences, aphasia and aging". En M. Gernsbacher (ed.), Handbook of Psycholinguistics. San Diego, C. A.: Academic Press, pp. 1075-1122.

Cerella, J. 1985. "Information processing rates in the elderly". En Psychologycal Bulletin, 98, pp. 67-83.

Cheung, H. \& Kemper, S. 1992. "Competing complexity metrics and adults production of complex sentences”. En Applied Psycholinguistics, 13, pp. 53-76.

Clifton, Ch., Traxler, M., Mohamed, M., Williams, R., Morris, R. \& Rayner, K. 2003. "The use of thematic role information in parsing: Syntactic processing autonomy revisited". En Journal of Memory and Language, 49, pp. 317-344.

Cuetos-Vega, F., Menéndez-González, M. y Calatayud-Noguera, T. 2007. "Descripción de un nuevo test para la detección precoz de la enfermedad de Alzheimer”. En REV. NEUROL, 44 (8), pp. 469-474.

Daneman, M. \& Carpenter, P. 1980. "Individual diferences in working memory and reading". En Journal of Verbal Learning and Verbal Behavior, 19, pp. 450466.

Daneman, M. \& Merikle, P. M. 1996. "Working memory and language comprehension: A meta-analysis". En Psychological Bulletin and Review, 3, pp. 422-433.

Davidson, D., Zacks, R. T. \& Ferreira, F. 2003. "Age preservation of the syntactic 
processor in production". En Journal of Psycholinguistic Research, 32, 5, pp. 541-566.

Del Río, D. y López-Higes, R. 2006. "Efectos de la memoria operativa y de una carga de procesamiento en la comprensión de oraciones". En Psicológica, 27, pp. 79-95.

Echeverría. M. y Urrutia, M. 2004. Incidencia del envejecimiento en el acceso léxico. En Revista Chilena de Fonoaudiología, 5 (2), pp. 7-23.

Escobar. A. 2001. "Envejecimiento cerebral normal". En Revista Mexicana de Neurociencia, 2(4), pp. 197-202.

Federmeier, K. D., McLennan, D. B., De Ochoa, E. \& Kutas, M. 2002. "The impact of semantic memory organization and sentence context in spoken language processing by younger and older adults: An ERP study". En Psychophysiology, 39, pp. 133-146.

Fernández-Ballesteros, R. 2005. "Vital Aging: A Psychosocial program for promoting optimal aging". En European Psychologist, 10, pp. 146-156.

Ferreira, F. \& Patson, N. D. 2007 "The 'good-enough' approach to language comprehension”. En Language and Linguistics Compass, 1 (1-2), pp. 71-83.

Gil, G. P. 2000. "Bases fisiológicas del envejecimiento cerebral". En Rev Mult Gerontol, 10(2), pp. 67-91.

Grady, Ch. 2001. "Age-Related Changes in the Functional Neuroanatomy of Memory”. En M. Naveh-Benjamin, M. Moscovitch \& H. L. Roediger (eds.), Perspectives on Human Memory and Cognitive Aging: Essays in Honour of Fergus Craik. New York: Psychology Press, pp. 325-333.

Grossman, M., A. Cooke, Ch. DeVita, D. Alsop, J. Detre, W. Chen \& J. Gee. 2002. "Age-related changes in working memory during sentence comprehension: An fMRI study". En NeuroImage, 15, pp. 302-317.

Hasher, L. \& Zacks, R. T. 1988. "Working memory, comprehension and aging: A review and a new view”. En G. H. Bower (ed.), The Psychology of Learning and Motivation, Vol. 22. New York: Academic Press, pp. 193-226.

Hasher, L., Lustig, C. \& Zacks, R. 2007. "Inhibitory mechanisms and the control of attention”. En A. Conway, C. Jarrold, M. Kane, A. Miyake \& J. Towse (eds.), Variation in working memory. New York: Oxford University Press, pp. 227-249.

James, L. E. \& MacKay, D. G. 2001. "H. M., word knowledge and aging: Supports for a new theory of long-term retrograde amnesia". En Psychological Sciences, 12, pp. 485-492.

James, L. E., Burke, D. M., Austin, A., \& Hulme, H. 1998. "Production and perception of 'verbosity' in younger and older adults". En Psychology and $A g$ ing, 13, pp. 355-367.

Juncos, O. 1998a. Lenguaje y envejecimiento: Bases para la intervención. Barcelona: Masson. 
Juncos, O. 1998b. "Problemas de acceso léxico en la vejez. Bases para la intervención", Anales de psicología 14(2):169-176.

Junqué, C. y Jódar, M. 1990. "Velocidad de procesamiento cognitivo en el envejecimiento". En Anales de Psicología, 6 (2), pp. 199-207.

Just, M. A. \& Carpenter, P. A. 1992. "A capacity theory of comprehension: Individual differences in working memory". En Psychological Review, 99, pp. 122149.

Kemmer, L., Coulson, S., De Ochoa, E. \& Kutas, M. 2004. "Syntactic processing with aging: A event-related potencial study". En Psychophysiology, 41, pp. 372-384.

Kemper, S. 1987. "Life-span changes in syntactic complexity". En Journal of Gerontology, 42, pp. 323-328.

Kemper, S. 1992. "Language and Aging”. En F. I. M. Craik \& T. Salthouse (eds.), The Handbook of Aging and Cognition. Hillsdale N. J.: Erlbaum, pp. 213270.

Kemper, S. \& Harden, T. 1999. "Experimentally disentangling what's beneficial about elderspeak from what's not". En Psychology and Aging, 14, pp. 656-670.

Kemper, S. \& Kemptes, K. 1999. "Limitations on syntactic processing". En S. Kemper \& R. Kliegl (eds.), Constraints on Language. Aging, Grammar and Memory. Norwell: Kluwer, pp. 79-106.

Kemper, S., Herman, R. \& Liu, Ch. 2004. "Sentence production by young and older adults in controlled contexts". En Journal of Gerontology: Psychological Science, 59B, pp. 230- 234.

Kemper S. \& Sumner, A. 2001. "The structure of verbal abilities in young an older Adults". En Psychology and Aging, 16, pp. 312-322.

Kemper S., Thompson, T. \& Marquis, J. 2001. "Longitudinal change in language production: Effects of aging and dementia on grammatical complexity and propositional content”. Psychology of Aging, 16, 4, pp. 600-614.

Kemper, S., Greiner, L. H., Marquis, J. G., Prenovost, K. \& Mitzner, T. L. 2001. "Language decline across the life span: Finding from the Nun Study". En Psychology and Aging, 16, pp. 227-239.

Kemptes, K. \& Kemper, S. 1997. "Younger and older adults' on-line processing of syntactically ambiguous sentences". En Psychology and Aging, 12, pp. 362371.

King, J. \& Just, M. A. 1991. "Individual differences in syntactic processing. The rol of working memory". En Journal of memory and language, 30, pp. 580602.

Kynette, D. \& Kemper, S. 1986. "Aging and the lost off grammatical forms: A cross sectional studio of language performance". En Language and Communications, 6, pp. 65-72.

Liberman, A. M., Cooper, F. S., Shankweiler, D. P. \& Studdert-Kennedy, M. 
1967. "Perception of the speech code". En Psychological Review, 74, pp. 43161.

Lindenberger, U. \& Baltes, P. B. 1997. "Intellectual functioning in old and very old age: Cross-sectional results from the Berlin Aging Study". En Psychology and Aging, 12, pp. 410-432.

MacKay, D. G. \& James, L. E. 2001. "The binding problem for syntax, semantics, and prosody: H. M. 's selective sentences-reading deficits and theoretical syndrome approach". En Language and Cognitive Processes, 16, pp. 419-460.

Madden, D. J. 1992. "Four to ten milliseconds per year: Age related slowing of visual word identificacion". En Journal of Gerontology: Psychological Sciences, 47, pp. 59-68.

Madden, D. J., Langley, L. K., Denny, L. L., Turkington, T. G., Provenzale, J. M. \& Hawk, T. C. 2002. "Adult age differences in visual word identification: Functional neuroanatomy by positron emission tomography". En Brian and Cognition, 49, pp. 297-321.

McDowd, J. 2001. "Levels of processing in selective attention and inhibition: Age differences and similarities". En M. Naveh-Benjamin, M. Moscovitch \& H. L. Roediger (eds.), Perspectives on Human Memory and Cognitive Aging: Essays in Honour of Fergus Craik. New York: Psychology Press, pp. 135-147.

McGuire, L. C., Morian, A., Goldding, R., \& Smyer, M. A. 2000. "Older adults' memory for medical information: Influence of elderspeak and note taking". En International Journal of Rehabilitation and Health, 5, pp. 117-128.

Metcalfe, J. \& Kornell, N. 2003. "The dinamics of learning and allocation of stydy time to a region of proximal learning". En Journal of Experimental Psychology: General, 132, pp. 530-542.

Miles, J. R. \& Stine-Morrow, E. A .L. 2004. "Adult age differences in self regulated learning in reading sentences". En Psychology and Aging, 19, pp. 626-636.

Nelson, T. O. 1996. "Consciousness and metacognition". En American Psychologist, 51, pp. 102-116.

Obler, L. K.; Fein, D.; Nicholas, M. \& Albert, M. L. 1991. "Auditory comprehension and aging: Decline in syntactic processing". En Applied Psycholinguistics, 12, pp. 433-452.

Park, D. C. 2000. "The basic mechanisms accounting for age-related decline in cognitive function”. En D. C Park,. \& N. Schwarz (eds). Cognitive Aging: A Primer. Philadelphia: Taylor and Francis Group, pp. 3-22.

Park, D. \& Payer, D. 2001. "Working Memory Across the Adult Lifespan". En E. Bialystok \& F. I. M. Craik (eds.), Lifespan cognition: Mechanisms of change. New York: Oxford University Press, pp. 128-142.

Payer, D., Marshuetz, CH., Sutton, B., Hebrank, A., Welsh, R. C. \& Park, D. 2006. "Decreased neural specialization in old adults on a working memory task". En NeuroRport, 5, pp. 487-491. 
Peronard, M. 1997. "Experiencia y conocimiento metacognitivos”. En Signos, 3839, pp. 121-131.

Peronard, M. 1999. "Metacognición y conciencia”. En G. Parodi (ed.), Ensayos en honor a Luis A. Gómez Macker. Valparaíso: Ediciones Universitarias de la Universidad Católica de Valparaíso, pp. 43-57.

Polk T. A. \& Farah M. J. 1998. "The neural development and organization of letter recognition: evidence from functional neuroimaging, computational modeling, and behavioral studies". En Proceedings of the National Academy of Sciences, 95, pp. 847-852.

Radvansky, G. A., Copeland, D. E., Berish, D. E., \& Dijkstra, K. 2003. "Aging and situation model updating". En Aging, Neuropsychology and Cognition, 10, pp. 158-166.

Radvansky, G. A., Zwaan, R. A., Curiel, J. M. \& Copeland, D. E. 2001. "Situations models and aging". En Psychology and Aging, 16, pp. 145-160.

Redolat, R. y Carrasco, M. C. 1998. “¿Es la plasticidad cerebral un factor crítico en el tratamiento de las alteraciones cognitivas asociadas al envejecimiento?”. En Anales de Psicología, 14, pp. 45-53.

Riffo, B. y Benoit, C. 2007. "Procesamiento de oraciones con incrustación central y derecha en adultos jóvenes y adultos mayores". RLA, 45 (1), pp. 13-31.

Riffo, B. y Véliz M. 2008. Informe final proyecto Fondecyt 1050591. Comisión Nacional de Ciencia y Tecnología (Conicyt), Chile.

Salazar, O. 2008. Marcadores discursivos y discurso senescente: análisis del habla producida por ancianos normales y ancianos diagnosticados con demencia tipo Alzheimer. Tesis Doctoral no publicada. Universidad de Valladolid.

Salthouse, T. A. 1996. "The processing-speed theory of adult age differences in cognition”. En Psychological Review, 103, pp. 403-428.

Salthouse, T. \& Craik, F. I. M. 2000. "Closing comments". En F. M. I. Craik \& T. A. Salthouse (eds.), The handbook of aging and cognition. Mahwah: N. J.: Erlbaum, pp. 689-703.

Salthouse, T. A., Hancock, H. E., Meinz, E. J. \& Hambrick, D. Z. 1996. "Interrelations of age, visual acuity, and cognitive functioning. En Journal of Gerontology, 51B, pp. 317-330.

Schneider, B. A. 2001. "Sensation, Cognition and Levels of Processing in Aging". En M. Naveh-Benjamin, M. Moscovitch \& H. L. Roediger (eds.), Perspectives on Human Memory and Cognitive Aging: Essays in Honour of Fergus Craik. New York: Psychology Press, pp. 298-313.

Schneider, B. A., Daneman, M. \& Pichora-Fuller, M. K. 2002. "Listening in aging adults: From discourse comprehension to psychoacoustics". En Canadian Journal of Experimental Psychology, 56, pp. 139-152.

Schneider, B. A. \& Pichora-Fuller, M. K. 2000. "Implications of perceptual deterioration for cognitive aging research". En F. I. M. Craik \& T. A. Salthouse 
(eds.), Handbook of aging and cognition. Hillside, N.J.: L. Erlbaum, pp. 155220.

Schneider, B. A. \& Pichora-Fuller, M. K. 2001. "Age-related changes in temporal processing: Implications for listening comprehension". En Seminars in Hearing, 22, pp. 227-239.

Singer, T., Verhaeghen, P., Ghisletta, P., Lindenberger, U. \& Baltes, P. B. 2003. "The fate of cognition in very old age: Six years longitudinal findings in the berlin Aging Study (BASE)”. En Psyhology and Aging, 18, pp. 318-331.

Smith, J. \& Baltes, P. 1996. "Altern aus psychologische Perspektive: Trends und Profile im hohen Alter”. En K. Mayer \& P. Baltes (eds.), Die Berliner Altersstudie. Berlin: Akademie Verlag, pp. 221-250.

Snowdon, D. A., Greiner, L., Kemper, S., Nanayyakara, N., \& Mortimer, J. A. 1999. "Linguistic ability in early life and longevity: Findings from the Nun Study”. En J. M. Robin, B. Forette, C. Francheschi, \& M. Allard (eds.), The paradoxes of longevity. Amsterdam: Springer, pp. 103-113.

Stine-Morrow, E. A. L. 2007. "The Dumbledore hypothesis of cognitive aging". En Current Directions in Psychological Science, 16, 6, pp. 295-299.

Stine-Morrow, E. A. L., Miller, L. \& Hertzog, C. 2006a. "Aging and self-regulated language processing". En Psychological Bulletin, 132, 4, pp. 582-606.

Stine-Morrow, E. A. L., Miller, L. \& Leno, R. 2001. "Aging resourse allocation to narrative text". En Aging Neuropsychology and Cognition, 8, pp. 36-53.

Stine-Morrow, E. A. L., Morrow, D. G. \& Leno, R. 2002. "Aging and the representation of spatial situations in narrative understanding". En Journals of Gerontology: Psychological Sciences, 57, pp. 91-97.

Stine-Morrow, E. A. L., Shake, M. C., Miles, J. R. \& Noh, S. R. 2006b. "Adult age differences in the effects of goals on self-regulated sentence processing". En Psychology and Aging, 21, 4, pp. 790-803.

Tapia, M. 2005. Producción de habla y fenómenos de vacilación en la conversación de adultos jóvenes y adultos mayores. En Anales de psiquiatría, Vol. 21, No 5: 215-222

Thiede, K. W. \& Dunlosky, J. 1999. "Toward a general model of self-regulated study: An analysis of selection of items for study and self-paced study time". En Journal of Experimental Psychology: Learning, Memory and Cognition, 25, pp. 1024-1137.

Thornton, R. \& Light, L. 2006. "Language comprehension and production in normal aging". En J. E. Birren \& K. W. Schaie (eds.), Handbook of the Psychology of Aging. New York, N.Y.: Academic Press, pp. 261-287.

Tun, P. A. \& Wingfield, A. 1999. "One voice to many: Adult age differences in language processing with different types of distracting sounds". En Journals of Gerontology: Psychologial Sciences, 54B, pp. 317-327.

Véliz, M. 2004. "Procesamiento de estructuras sintácticas complejas en adultos 
mayores y adultos jóvenes". En Estudios Filológicos, 39, pp 65-81.

Véliz, M., Riffo, B. y Vásquez, A. 2009. "Recuerdo inmediato de oraciones de sintaxis compleja en adultos jóvenes y mayores". En evaluación para Estudios Filológicos.

Vitevitch, M. S. \& Sommers, M. S. 2003. "The facilitative influence of phonological similarity and neighbordhood frecuency in speech production in younger and older adults". En Memory \& Cognition, 31, pp. 491-504.

Waters, G. \& Caplan, D. 1999. "Verbal working memory capacity and on-line sentence processing efficiency in the elderly". En S. Kemper \& R. Kliegl (eds.), Constraints on Language. Aging, Grammar and Memory. Boston: Kluwer Academic Publishers, pp. 107-136.

Waters, G. \& Caplan, D. 2001. "Age, working memory and on-line syntactic processing in sentence comprehension". En Psychology and Aging, 16, pp. 128144.

Waters, G., Caplan, D, Alpert, N. \& Stanczak, L. 2003. “Individual differences in rCBF correlates of syntactic processing in sentence comprehension: effects of working memory and speed of processing". En NeuroImage, 19, pp. 101-112.

Wingfield, A. \& Stine-Morrow, E. A. L. 2000. "Language and Speech". En F. I. M. Craik \& T. A. Salthouse (eds.), Handbook of aging and cognition. Hillside, N.J.: L. Erlbaum, pp. 293-357.

Wittig, F. 2004. Estructura narrativa en el discurso oral de adultos mayores. En Rev. Signos, vol. 37, (56), pp. 91-101.

Zacks, R. \& Hasher, L. 1997. "Cognitive gerontology and attentional inhibition: A reply to Burke and McDowd”. En Journal of Gerontology: Psychological Sciences, 52B, pp. 274-283.

Zacks, R., Hasher, L. \& Li, K. Z. H. 2000. "Human memory". En F. I. M. Craik $\&$ T. A. Salthouse (eds.), Handbook of aging and cognition. Hillside, N.J.: L. Erlbaum, pp. 293-357. 